\title{
Cetuximab for the treatment of locally advanced and recurrent/metastatic oral cancer: An investigation of distant metastasis
}

\author{
TOMOFUMI NARUSE ${ }^{1}$, SOUICHI YANAMOTO ${ }^{1}$, YUKI MATSUSHITA $^{1}$, YUKI SAKAMOTO ${ }^{1}$, KOTA MORISHITA ${ }^{1}$, \\ SEIGO OHBA $^{2}$, TAKESHI SHIRAISHI ${ }^{2}$, SHIN-ICHI YAMADA ${ }^{3}$, IZUMI ASAHINA ${ }^{2}$ and MASAHIRO UMEDA ${ }^{1}$ \\ Departments of ${ }^{1}$ Clinical Oral Oncology and ${ }^{2}$ Regenerative Oral Surgery, Graduate School of Biomedical Sciences, \\ Nagasaki University Hospital, Nagasaki, Nagasaki 852-8588; ${ }^{3}$ Department of Dentistry and Oral Surgery, \\ Shinshu University School of Medicine, Matsumoto, Nagano 390-8621, Japan
}

Received November 26, 2015; Accepted March 21, 2016

DOI: $10.3892 / \mathrm{mco} .2016 .928$

\begin{abstract}
The aim of this retrospective study was to assess the efficacy and safety of cetuximab therapy for patients with locally advanced (LA) and recurrent/metastatic (R/M) oral squamous cell carcinoma (OSCC), with a specific focus on distant metastases (DMs). Data from 21 patients with unresectable LA and R/M OSCC treated with cetuximab therapy in our department between December, 2012 and July, 2015 were reviewed. The endpoint was the time-to-progression and the assessments made were tumor response rate, progression-free survival (PFS), overall survival (OS) and safety. The overall response rate was $57.1 \%$, with a complete response (CR) rate of 33.3\%. The overall median PFS and OS were 5.5 and 8.0 months, respectively. For patients with DMs, the overall response rate was $60.0 \%$, with a CR rate of $40.0 \%$. The median PFS and OS were 3.8 and 5.8 months, respectively. In addition, improved 1-year OS was observed following approval of cetuximab, although the differences between the group of patients treated after that time and historical controls were not statistically significantly $(\mathrm{P}=0.246)$. Grade $3-4$ adverse events included infusion reaction (4 cases), neutropenia, hypophosphatemia,
\end{abstract}

Correspondence to: Dr Tomofumi Naruse, Department of Clinical Oral Oncology, Graduate School of Biomedical Sciences, Nagasaki University Hospital, 1-7-1 Sakamoto, Nagasaki, Nagasaki 852-8588, Japan

E-mail: naruse12@nagasaki-u.ac.jp

Abbreviations: CI, confidence interval; CR, complete response; CT, computed tomography; DM, distant metastasis; DSS, disease-specific survival; EGFR, epidermal growth factor receptor; HNSCC, head and neck squamous cell carcinoma; LA, locally advanced; R/M, recurrent/metastatic; OS, overall survival; OSCC, oral squamous cell carcinoma; PD, progressive disease; PFS, progression-free survival; PR, partial response; SCC, squamous cell carcinoma; SD, stable disease; PS, performance status

Key words: oral cancer, cetuximab, distant metastasis, recurrent/metastatic, infusion reaction upper gastrointestinal hemorrhage, liver toxicity and mucositis (1 case each). There was one cetuximab-related death due to interstitial pneumonia. An acne-like rash was observed in all cases, but no grade 3 or 4 rash was reported. Hypomagnesemia was observed in 10 cases. Our results suggest that cetuximab may display significant therapeutic efficacy in patients with unresectable LA and R/M OSCC, including those with DMs.

\section{Introduction}

Oral squamous cell carcinoma (OSCC) represents 2-3\% of all human cancers and is the 6th most frequent type of cancer worldwide $(1,2)$. OSCC has a consistently poor prognosis and remains a lethal disease in $>50 \%$ of cases diagnosed annually (3). The current management and treatment for the majority of OSCC patients is surgery, and postoperative concurrent chemoradiotherapy is a widely accepted standard of care for high-risk OSCC (4). This treatment strategy has led to significant improvements in locoregional control and disease-free survival, but not in overall survival (OS) (5). The risk factors associated with lack of improvement in OS remain unclear, but one risk factor has been hypothesized to be distant metastasis (DM). In the head and neck region, it was reported that the hypopharynx and supraglottis were the sites with a higher risk of DM (9.4 and $8.9 \%$, respectively), whereas the oral cavity had a lower risk (3.2\%) (6). It has been reported that DMs occur 1-76 months after radical surgery in $\sim 10 \%$ of patients with OSCC (7). As DMs reduce the patients' quality of life and carry a poor prognosis, improved treatment of DMs is an important consideration.

Cetuximab, an epidermal growth factor receptor (EGFR) inhibitor, was approved for the treatment of locally advanced (LA) and recurrent/metastatic (R/M) head and neck squamous cell carcinoma (HNSCC) in December, 2012 in Japan. Phase III trials demonstrated that cetuximab used in combination with radiotherapy in LA HNSCC (8) and in combination with platinum-based chemotherapy as first-line treatment for R/M HNSCC (9) achieved a higher response rate and a significant increase in OS. In addition, it has been reported that cetuximab used in combination with paclitaxel 
is effective when platinum-based chemotherapy fails in $\mathrm{R} / \mathrm{M}$ HNSCC $(10,11)$. However, the efficacy of cetuximab for DMs remains unclear.

The aim of this retrospective study was to assess the efficacy and safety of cetuximab therapy in patients with LA and $\mathrm{R} / \mathrm{M}$ OSCC, with a specific focus on patients with DMs.

\section{Patients and methods}

Study design. We retrospectively reviewed records of patients with confirmed unresectable LA and R/M OSCC who were treated with cetuximab between December, 2012 and July, 2015 (cetuximab group). This study was approved by the independent Ethics Committee of our Nagasaki University Hospital. The endpoint of this trial was defined as the time-to-disease progression, and we examined the tumor response rate, progression-free survival (PFS), OS and safety. Tumor response was assessed every 4-8 weeks with repeated clinical and enhanced computed tomography (CT) assessments, until progressive disease (PD) was observed; the best overall response was evaluated according to the Response Evaluation Criteria in Solid Tumours for the duration of treatment (12). PFS was defined as the time from the date of the first cetuximab administration to the date of PD or relapse, whichever occurred first. OS was defined as the time from the date of cetuximab administration to the date of death. Survival distributions were calculated with the Kaplan-Meier method and compared using the log-rank test. Two-sided confidence intervals (CIs) were calculated according to Clopper and Pearson. Toxic effects were evaluated according to the National Cancer Institute-Common Terminology Criteria for Adverse Events, version 4.0 (13).

Treatment. The regimens used in our department were cetuximab plus radiotherapy according to the Bonner trial (8), cetuximab plus cisplatin and 5-fluorouracil according to the EXTREME trial (9), and cetuximab plus paclitaxel $(10,11)$. Prior to drug administration, the serum levels of surfactant protein-A, surfactant protein-D and Krebs von den Lungen-6 were assessed using blood tests; plain radiography and $\mathrm{CT}$ of the chest were performed to determine the presence of interstitial pneumonia. In addition, the serum levels of tick, mammalian meat and flatfish antibodies were assessed for allergy and specific IgG antibody titres. Cetuximab was administered at a dose of $400 \mathrm{mg} / \mathrm{m}^{2}$ for the first injection and $250 \mathrm{mg} / \mathrm{m}^{2}$ weekly thereafter. A total radiation dose of 60-66 Gy was administered once daily in fractions of 1.8-2.0 Gy, with five fractions administered per week. Cisplatin was administered at a dose of $100 \mathrm{mg} / \mathrm{m}^{2}$ on day 1 , and 5-fluorouracil was administered at a dose of $1,000 \mathrm{mg} / \mathrm{m}^{2}$ for 4 days. Paclitaxel was administered weekly at a dose of $60-80 \mathrm{mg} / \mathrm{m}^{2}$. Dosage was decided according to the patient's general condition. Patients who had at least stable disease (SD) received cetuximab therapy until $\mathrm{PD}$ or unacceptable toxic effects occurred.

\section{Results}

Patient characteristics. The patient clinicopathological characteristics are summarized in Table I. Over a period of 2 years and 6 months, 21 patients were enrolled in the study; $47.6 \%$ of the

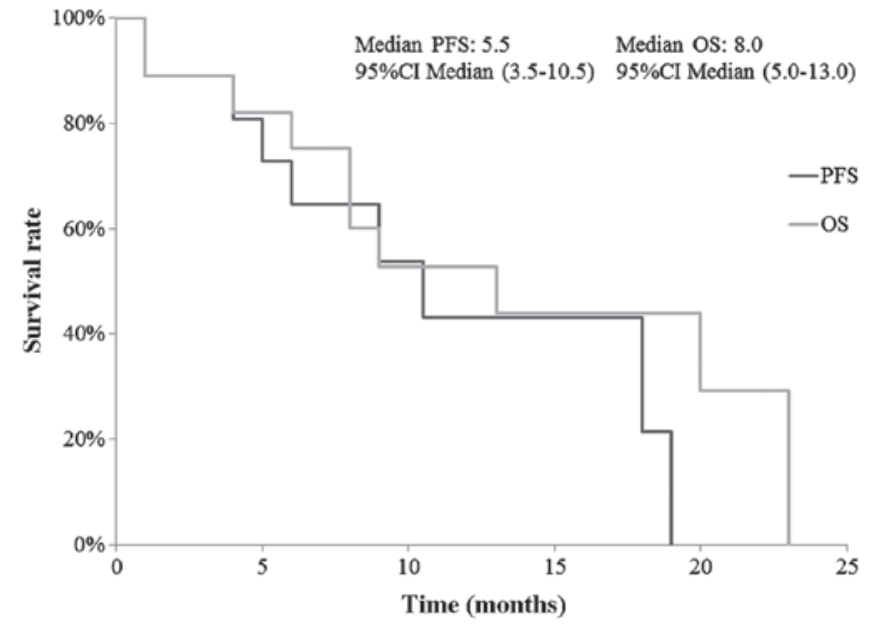

Figure 1. Kaplan-Meier estimates of progression-free survival (PFS) and overall survival (OS). CI, confidence interval.

patients were male and $52.4 \%$ were female, and 20 patients had a performance status of 0 or 1 . The median age of the patients was 73 years, (range, 51-88 years). The most common site of the primary tumour was the mandibular gingiva (52.4\%), followed by the tongue (23.8\%) and maxillary gingiva (9.52\%). On pathological examination, 16 of the 21 tumors were SCCs $(76.2 \%)$ and the remaining 5 were adenosquamous cell carcinoma $(\mathrm{n}=1$, $4.76 \%)$, ameloblastic carcinoma $(n=1,4.76 \%)$, myoepithelial carcinoma $(\mathrm{n}=1,4.76 \%)$, undifferentiated carcinoma $(4.76 \%)$ and unclear $(n=1,4.76 \%)$. The overexpression of EGFR was confirmed by immunostaining in each of the 5 non-SCC cases prior to drug administration. There were 3 patients with LA carcinoma (14.3\%) and 18 with R/M carcinoma (85.7\%). A total of 18 patients had undergone previous therapy: 8 had undergone surgery alone (38.1\%), 2 had undergone surgery and adjuvant radiotherapy (9.5\%), and 8 had undergone surgery and adjuvant chemoradiotherapy (38.1\%). With respect to initial treatment regimens, of the 21 tumors, 9 had been treated with cetuximab plus radiotherapy (42.8\%), 9 with cetuximab plus paclitaxel (42.8\%), 2 with cetuximab plus cisplatin and 5-fluorouracil $(9.5 \%)$ and 1 with cetuximab alone (4.8\%). The median number of treatment courses with cetuximab was 9 (range, 0-70).

Efficacy. The details of all cases and of DM cases are presented in Table II. The overall response rate was $57.1 \%$ (95\% CI: 33.7-78.2\%), with a complete response (CR) rate of $33.3 \%$ (95\% CI: $13.9-56.9 \%$ ) and a partial response (PR) rate of 23.8\% (95\% CI: 7.7-47.6\%). The disease control rate (PR plus SD) was $66.7 \%$ (95\% CI: 42.7-85.4\%). Cetuximab-refractory tumors were observed in 2 patients. Although 5 patients initially achieved disease control, they developed PD. Among these, 2 patients presented with brain metastasis during cetuximab administration, although 1 achieved locoregional and lung metastasis control. The 1-year PFS and OS were 43.1 and $52.7 \%$, respectively. The median PFS and OS were 5.5 months (95\% CI: 3.5-10.5 months) and 8.0 months (95\% CI: 5-13 months), respectively (Fig. 1).

A total of 10 patients treated with cetuximab therapy had DMs. The most common distant site was the lung $(70.0 \%)$, followed by the upper mediastinal lymph nodes 
Table I. Clinicopathological characteristics of the 21 patients.

\begin{tabular}{|c|c|}
\hline Characteristics & No. of cases $(\%$ \\
\hline \multicolumn{2}{|l|}{ Gender } \\
\hline Male & $10(47.6)$ \\
\hline Female & $11(52.4)$ \\
\hline \multicolumn{2}{|l|}{ Age, years } \\
\hline Range & $51-88$ \\
\hline Median & 73 \\
\hline \multicolumn{2}{|l|}{ Primary site } \\
\hline Tongue & $5(23.8)$ \\
\hline Mandibular gingiva & $11(52.4)$ \\
\hline Maxillary gingiva & $2(9.52)$ \\
\hline Oral cavity floor & $1(4.76)$ \\
\hline Intraosseous & $1(4.76)$ \\
\hline Unknown & $1(4.76)$ \\
\hline \multicolumn{2}{|l|}{ Pathological diagnosis } \\
\hline SCC & $16(76.2)$ \\
\hline Ameloblastic carcinoma & $1(4.76)$ \\
\hline Myoepithelial carcinoma & $1(4.76)$ \\
\hline Adeno SCC & $1(4.76)$ \\
\hline Undifferentiated carcinoma & $1(4.76)$ \\
\hline Unclear & $1(4.76)$ \\
\hline \multicolumn{2}{|l|}{ Performance status score } \\
\hline 0 & $11(52.4)$ \\
\hline 1 & $9(42.9)$ \\
\hline 2 & $1(4.8)$ \\
\hline \multicolumn{2}{|l|}{ Pattern of disease } \\
\hline LA & $3(14.3)$ \\
\hline $\mathrm{R} / \mathrm{M}$ & $18(85.7)$ \\
\hline \multicolumn{2}{|l|}{ Previous treatment } \\
\hline Surgery alone & $8(44.4)$ \\
\hline Surgery + adjuvant RT & $2(11.1)$ \\
\hline Surgery + adjuvant CCRT & $8(44.4)$ \\
\hline \multicolumn{2}{|l|}{ Initial treatment } \\
\hline Cet + RT & $9(42.8)$ \\
\hline Cet + TXL & $9(42.8)$ \\
\hline $\mathrm{Cet}+\mathrm{FP}$ & $2(9.5)$ \\
\hline Cet alone & $1(4.8)$ \\
\hline \multicolumn{2}{|l|}{ Number of treatment cycles } \\
\hline Range & $0-70$ \\
\hline Median & 9 \\
\hline
\end{tabular}

SCC, squamous cell carcinoma; LA, locally advanced; R/M, recurrent/metastatic; RT, radiotherapy; CCRT, concurrent chemoradiotherapy; Cet, cetuximab; TXL, paclitaxel; FP, cisplatin and 5-fluorouracil.

(10.0\%), parapharyngeal lymph nodes $(10.0 \%)$, and multiple bone metastases on the ribs, iliac bone and vertebrae $(10.0 \%)$. The overall response rate among patients with DMs was $60.0 \%$ (95\% CI: $26.2-87.8 \%$ ), with a CR rate of $40.0 \%(95 \% \mathrm{CI}: 12.2-73.8 \%)$ and a PR rate of $20.0 \%$

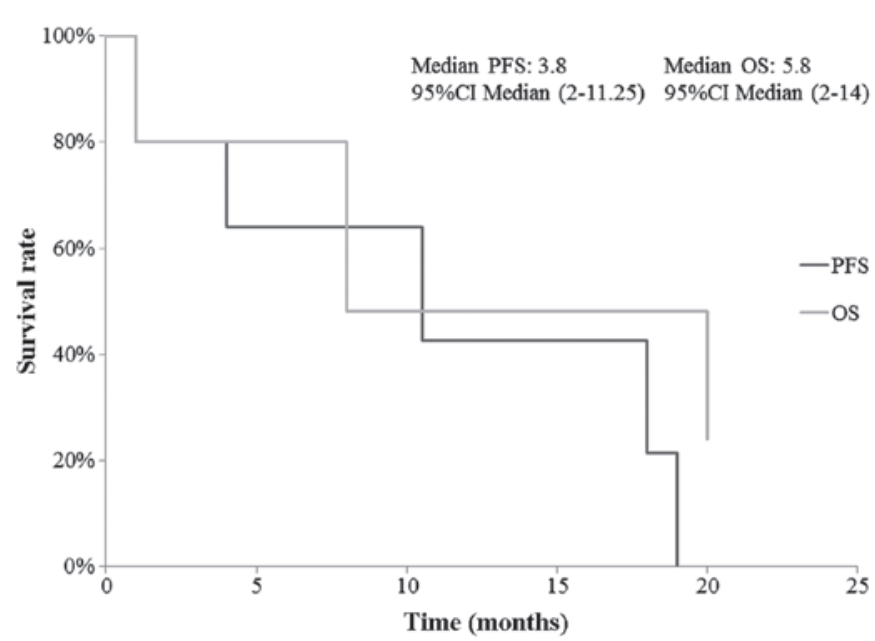

Figure 2. Kaplan-Meier estimates of progression-free survival (PFS) and overall survival (OS) in patients with distant metastases. CI, confidence interval.

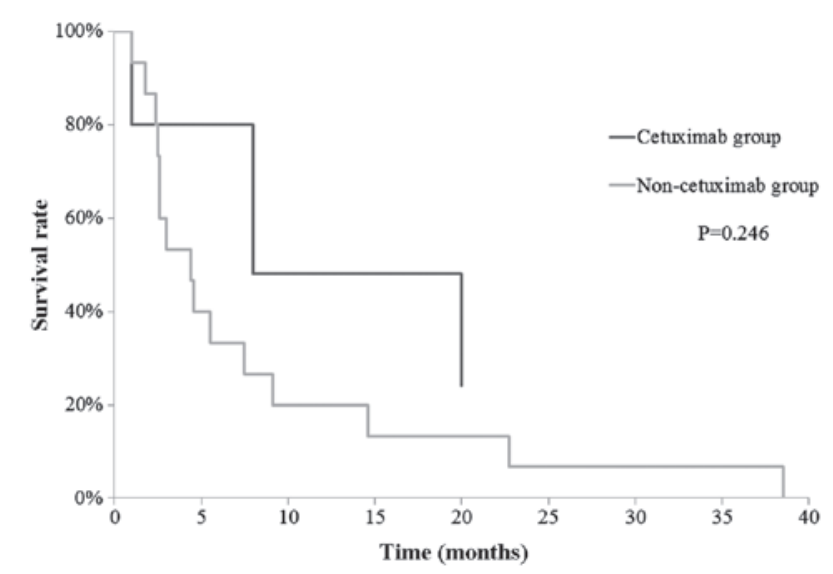

Figure 3. Kaplan-Meier estimates of progression-free survival (PFS) and overall survival (OS) according to treatment group (cetuximab vs. non-cetuximab). CI, confidence interval.

(95\% CI: $2.5-55.6 \%$ ). The disease control rate was $70.0 \%$ (95\% CI: 34.8-93.8\%). No difference in the efficacy of cetuximab therapy was observed between different DM sites. The 1-year PFS and OS were 42.7 and $48.0 \%$, respectively. The median PFS and OS were 3.8 months (95\% CI: 2-11.25 months) and 5.8 months (95\% CI: 2-14 months), respectively (Fig. 2). In addition, we retrospectively reviewed the records of patients with confirmed DM between April, 2001 and November, 2012 before cetuximab was approved (non-cetuximab group), and a historical-control study was performed to compare the OS between the cetuximab and non-cetuximab groups in patients with DMs. The 1-year OS for each group was 48.0 and $20.0 \%$, respectively. No significant difference was observed $(\mathrm{P}=0.246)$, but the data indicated that cetuximab provided additional benefits in patients with DMs (Fig. 3).

Safety. Treatment-related grade 3-5 adverse events were reported in 9 patients $(42.9 \%$ ) (Table III). There were 3 grade 3 infusion reactions (anaphylaxis and dyspnea in all cases), 1 grade 4 reaction (anaphylaxis) and 1 grade 5 reaction (interstitial pneumonia) among patients receiving 
Table II. Tumor response.

\begin{tabular}{|c|c|c|c|c|}
\hline \multirow[b]{2}{*}{ Tumor response } & \multicolumn{2}{|c|}{ All cases } & \multicolumn{2}{|c|}{ DM cases } \\
\hline & $\mathrm{n}=21(\%)$ & $95 \% \mathrm{CI}$ & $\mathrm{n}=10(\%)$ & $95 \% \mathrm{CI}$ \\
\hline \multicolumn{5}{|l|}{ Best response } \\
\hline $\mathrm{CR}$ & $7(33.3)$ & $13.9-56.9$ & $4(40.0)$ & $12.2-73.8$ \\
\hline PR & $5(23.8)$ & $7.7-47.6$ & $2(20.0)$ & $2.5-55.6$ \\
\hline SD & $4(19.0)$ & $4.9-42.5$ & $2(20.0)$ & $2.5-55.6$ \\
\hline $\mathrm{PD}$ & $2(9.5)$ & $0.7-31.6$ & $2(20.0)$ & $2.5-55.6$ \\
\hline $\mathrm{NE}$ & $3(14.3)$ & $3.0-36.5$ & $4(40.0)$ & $12.2-73.8$ \\
\hline Overall response rate $(\mathrm{CR}+\mathrm{PR})$ & $12(57.1)$ & $33.7-78.2$ & $6(60.0)$ & $26.7-87.8$ \\
\hline Disease control rate $(\mathrm{CR}+\mathrm{PR}+\mathrm{SD})$ & $16(76.2)$ & $52.7-91.8$ & $8(80.0)$ & $43.2-98.5$ \\
\hline
\end{tabular}

CR, complete response; PR, partial response; SD, stable disease; PD, progressive disease; NE, not evaluable; CI, confidence interval.

Table III. Adverse events.

\begin{tabular}{|c|c|c|c|c|}
\hline Adverse events & Cet+RT $(n=9)$ & Cet+TXL (n=9) & Cet+FP $(n=2)$ & Cet $(n=1)$ \\
\hline \multicolumn{5}{|l|}{ Grade $\geq 3$} \\
\hline Infusion reaction & 1 & 3 & & \\
\hline Neutropenia & & 1 & & \\
\hline Hypophosphatemia & 1 & & & \\
\hline Upper gastrointestinal hemorrhage & & 1 & & \\
\hline Interstitial pneumonia & 1 & & & \\
\hline Liver toxicity & & 1 & & \\
\hline Mucositis & 1 & & & \\
\hline Diarrhea & & & 1 & \\
\hline \multicolumn{5}{|l|}{ Grade $1-2$} \\
\hline Acne-like rash & 7 & 8 & 2 & 1 \\
\hline Nausea & & & 2 & \\
\hline Paronychia & & 2 & & \\
\hline Peripheral neuropathy & & 2 & & \\
\hline Hypomagnesemia & 4 & 5 & 1 & \\
\hline Alopecia & & 1 & & \\
\hline Taste dysfunction & 3 & & & \\
\hline
\end{tabular}

Cet, cetuximab; RT, radiotherapy; TXL, paclitaxel; FP, cisplatin and 5-fluorouracil.

cetuximab, who had to withdraw from therapy. Grade 3 hypophosphatemia, neutropenia, upper gastrointestinal hemorrhage and liver toxicity were reported with the cetuximab plus paclitaxel regimen; grade 3 diarrhea was reported with the cetuximab plus cisplatin and 5-fluorouracil regimen; and grade 3 hypophosphatemia was reported with the cetuximab plus radiotherapy regimen, which led to the discontinuation of these drug combinations. Skin reactions, including an acne-like rash and paronychia, were seen in all patients; 11 patients had grade 1 (52.4\%), 7 had grade $2(33.3 \%)$, and 3 patients $(14.3 \%)$ had non-evaluable infusion-related reactions; no patient had an infusion-related reaction of grade $>3$. Grade 1-2 treatment-related adverse events included hypomagnesemia (47.6\%), taste dysfunction $(14.3 \%)$, alopecia $(9.5 \%)$, peripheral neuropathy $(9.5 \%)$ and nausea $(9.5 \%)$.

Case report. A representative case report in which $\mathrm{CR}$ was achieved with cetuximab therapy for a DM is shown. A 60-year old man was diagnosed with SCC of the mandible (T4NOM0, stage IVA) and underwent segmental mandibulotomy, modified radical neck dissection and reconstruction with fibular osteocutaneous free flaps. However, a DM occurred in the lung fields 10 months after surgery. As the patient experienced failure of a platinum-based chemoradiotherapy, cetuximab plus paclitaxel was administered (Fig. 4A and B). Following 

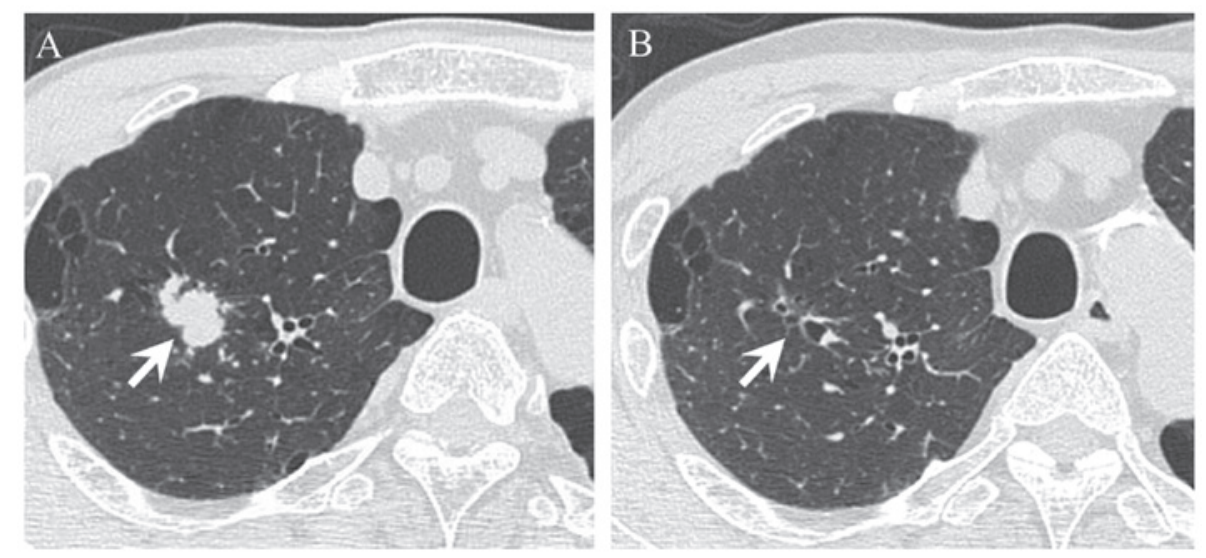

Figure 4. (A) Axial chest computed tomography detected multiple pulmonary nodules 10 months after surgery (arrow). (B) The nodules could not be detected 3 months after cetuximab administration.

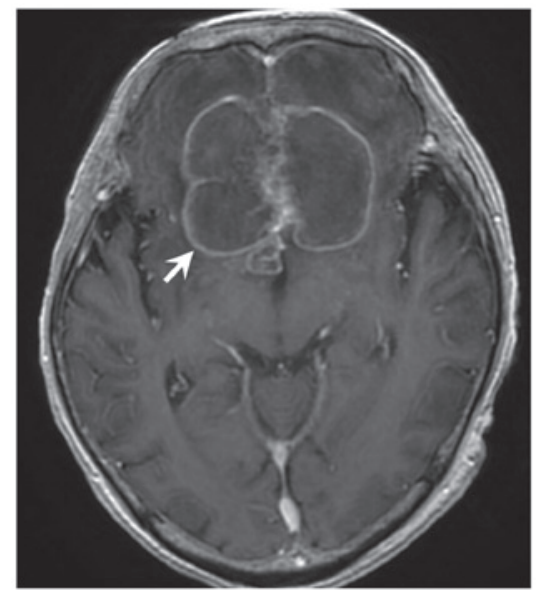

Figure 5. Gadolinium-enhanced T1-weighted magnetic resonance imaging of the head showing a $62 \times 40 \times 40-\mathrm{mm}$ ring-enhanced mass lesion in the frontal lobe bilaterally.

administration of 57 courses, brain metastases were detected by enhanced magnetic resonance imaging, despite better locoregional and lung DM control (Fig. 5). Palliative surgery for the brain metastases was performed at the Department of Neurosurgery, and metastasis of oral SCC to the brain was pathologically diagnosed. Following surgery, best supportive care was provided at another hospital.

\section{Discussion}

Further investigation of the effects of cetuximab therapy on OSCC may allow identification of new treatment possibilities, particularly with respect to DM. Therefore, the aim of the present study was to assess the efficacy and safety of cetuximab therapy in patients with LA and R/M OSCC in our department, with a specific focus on patients with DMs.

EGFR is overexpressed in 80-90\% of HNSCC cells, and its activation acts as a prognostic indicator, predicting poor survival and/or more advanced disease stage, promotion of tumor motility and invasiveness (14). Agra et al reported that patients whose tumours did not express EGFR had better treatment results following salvage surgery, with a disease-specific survival (DSS) rate of $64.3 \%$ at 3 years, while those whose tumours expressed EGFR had a DSS rate of $27.2 \%$ at 3 years (15). A phase III trial of the Radiation Therapy Oncology Group indicated that EGFR expression, which varied considerably among HNSCCs, was a strong independent prognostic indicator for OS and disease-free survival and a robust predictor of locoregional relapse, and that blockade of EGFR signaling sensitizes cells to the effects of radiation (16). Moreover, cetuximab represents a promising growth-inhibitory agent that may affect cellular proliferation, apoptosis and chemoradiosensitivity in SCC cell lines of the head and neck in vitro $(17,18)$. From these reports, inhibition of EGFR has shown strong clinical evidence of improving clinical outcome. The results of our study confirm its therapeutic efficiency in OSCC patients, with an overall response rate of $52.4 \%$, a CR rate of $28.6 \%$ and a median OS of 7.0 months. In cases with DMs, cetuximab therapy achieved an overall response rate of $50.0 \%$, a CR rate of $40.0 \%$ and a median OS of 8.0 months. Specifically, cetuximab therapy was shown to result in improved 1-year OS compared to the 1-year OS of the non-cetuximab therapy group using a historical-control study. Although the evidence was weak due to the non-uniform regimens in our study, the observed clinical outcomes were comparable to those reported by previous studies $(9-11,19)$. However, 5 patients experienced PD, and 2 patients presented with brain metastasis during cetuximab administration. Distant brain metastases from oral SCC are extremely rare, and it has been reported that brain metastases comprise $0.4-5.5 \%$ of all DMs (7,20-22). In brain metastases from HNSCC, it has been reported that human papillomavirus-positive HNSCCs are more prone to metastasize to distant and unusual sites $(22,23)$. However, it has been reported that adding cetuximab to radiotherapy plus cisplatin significantly prolonged the PFS and OS in human papillomavirus-positive HNSCC (24). Although it currently remains unclear, the association between cetuximab and brain metastasis may be associated with the re-overexpression of the human papillomavirus-related gene, which was once decreased by cetuximab.

Although cetuximab is expected to provide significant therapeutic benefits, infusion reactions have been reported during the administration or post-administration of monoclonal antibodies. The incidence of all-grade and grade 3-4 infusion reactions has been reported to be 13.5 and $2.9 \%$, respectively, in the Bonner et al trial (8), and 10 and 2.3\%, respectively, in 
the EXTREME trial (9), whereas Touma et al reported rates of 19.3 and $6.6 \%$, respectively (25). Due to these reasons, it has been reported the $\operatorname{IgE}$ antibodies were shown to be specific for the oligosaccharide galactose- $\alpha-1,3$-galactose ( $\alpha$-gal), which is present on the Fab portion of the cetuximab heavy chain (26), and $\operatorname{IgE}$ antibody formation against $\alpha$-gal is associated with tick bites and ingestion of mammalian meat $(27,28)$. In our study, the incidence of grade 3-4 infusion reactions was highly comparable to that reported by previous studies, but the association with allergy to $\alpha$-gal was negative. Interstitial pneumonia has been reported to be a rare adverse event of cetuximab (29). The incidence of all-grade and grade 3-4 infusion reactions has been reported to be 1.2 and $0.7 \%$, respectively, and 10 patients succumbed to this condition in a Japanese post-marketing surveillance of cetuximab in patients with metastatic colorectal cancer (30). In our study, one patient succumbed to acute respiratory distress syndrome, which occurred due to an interstitial pneumonia flare-up. Cetuximab-induced lung disease was diagnosed based on the clinical course and findings. Regarding the representative adverse events observed in our study, a grade 3-4 acne-like rash was not observed, but grade 3 hypophosphatemia, neutropenia, upper gastrointestinal hemorrhage and liver toxicity were observed. Although neutropenia is a known adverse event of cetuximab, hypophosphatemia, upper gastrointestinal hemorrhage and liver toxicity have not been reported in other HNSCC studies with cetuximab $(8-11,31)$. Most of these adverse events, including neutropenia, were observed with the cetuximab plus paclitaxel regimen. Hitt et al reported that grade 3-4 adverse events were observed in 30 of 46 patients (65\%), including an acne-like rash (24\%), neutropenia (13\%), and neuropathy/paresthesia (11\%) (10); and Péron et al reported that 20 of 42 patients $(48 \%)$ received a dose reduction or cessation of treatment after dose reduction (11). In our study, 6 of 9 patients $(66.6 \%)$ received dose reduction or discontinued treatment due to severe adverse events, possibly attributed to an interaction of paclitaxel and cetuximab.

To date, there are few treatment options for patients with DM following failure of platinum-containing chemotherapy. Our study suggests that cetuximab is expected to have significant therapeutic efficiency in patients with unresectable LA and R/M OSCC, including in those with DMs, although unacceptable or severe adverse events may often occur.

\section{References}

1. Jemal A, Bray F, Center MM, Ferlay J, Ward E and Forman D: Global cancer statistics. CA Cancer J Clin 61: 69-90, 2011.

2. Warnakulasuriya S: Global epidemiology of oral and oropharyngeal cancer. Oral Oncol 45: 309-316, 2009.

3. Rogers SN, Brown JS, Woolgar JA, Lowe D, Magennis P, Shaw RJ, Sutton D, Errington D and Vaughan D: Survival following primary surgery for oral cancer. Oral Oncol 45: 201-211, 2009.

4. Bernier J, Cooper JS, Pajak TF, van Glabbeke M, Bourhis J, Forastiere A, Ozsahin EM, Jacobs JR, Jassem J, Ang KK and Lefèbvre JL: Defining risk levels in locally advanced head and neck cancers: A comparative analysis of concurrent postoperative radiation plus chemotherapy trials of the EORTC (\#22931) and RTOG (\#9501). Head Neck 27: 843-850, 2005.

5. Cooper JS, Zhang Q, Pajak TF, Forastiere AA, Jacobs J, Saxman SB, Kish JA, Kim HE, Cmelak AJ, Rotman M, et al: Long-term follow-up of the RTOG 9501/intergroup phase III trial: Postoperative concurrent radiation therapy and chemotherapy in high-risk squamous cell carcinoma of the head and neck. Int J Radiat Oncol Biol Phys 84: 1198-1205, 2012.
6. Lim JY, Lim YC, Kim SH, Kim JW, Jeong HM and Choi EC: Predictive factors of isolated distant metastasis after primary definitive surgery without systemic treatment for head and neck squamous cell carcinoma. Oral Oncol 46: 504-508, 2010.

7. Takahashi M, Aoki T, Nakamura N, Carreras J, Kajiwara H, Kumaki N, Inomoto C, Ogura G, Kikuchi T, Kikuti YY, et al: Clinicopathological analysis of 502 patients with oral squamous cell carcinoma with special interest to distant metastasis. Tokai J Exp Clin Med 39: 178-185, 2014.

8. Bonner JA, Harari PM, Giralt J, Azamia N, Shin DM, Cohen RB, Jones CU, Sur R, Raben D, Jassem J, et al: Radiotherapy plus cetuximab for squamous-cell carcinoma of the head and neck. N Engl J Med 354: 567-578, 2006.

9. Vermonken JB, Mesia R, Rivera F, Remenar E, Kawecki A, Rottey S, Erfan J, Zabolotnyy D, Kienzer HR, Cupissol D, et al: Platinum-based chemotherapy plus cetuximab in head and neck cancer. N Engl J Med 359: 1116-1127, 2008.

10. Hitt R, Irigoyen A, Cortes-Funes H, Grau JJ, García-Sáenz JA and Cruz-Hernandez JJ; Spanish Head and Neck Cancer Cooperative Group (TTCC): Phase II study of the combination of cetuximab and weekly paclitaxel in the first-line treatment of patients with recurrent and/or metastatic squamous cell carcinoma of head and neck. Ann Oncol 23: 1016-1022, 2012.

11. Péron J, Ceruse P, Lavergne E, Buiret G, Pham BN, Chabaud S, Favier B, Girodet D, Zrounba P, Ramade A and Fayette J: Paclitaxel and cetuximab combination efficiency after the failure of a platinum-based chemotherapy in recurrent/metastatic head and neck squamous cell carcinoma. Anticancer Drug 23: 996-1001, 2012.

12. Eisenhauer EA, Therasse P, Bogaerts J, Schwartz LH, Sargent D, Ford R, Dancey J, Arbuck S, Gwyther S, Mooney M, et al: New response evaluation criteria in solid tumours: Revised RECIST guideline (version 1.1). Eur J Cancer 45: 228-247, 2009.

13. Eisenhauer EA, Therasse P, Bogaerts J, Schwartz LH, Sargent D, Ford R, et al: New response evaluation criteria in solid tumours: Revised RECIST guideline (version 1.1). Eur J Cancer 45: 228-247, 2009.

14. Laskin JJ and Sandler AB: Epidermal growth factor receptor: A promising target in solid tumours. Cancer Treat Rev 30: 1-17, 2004.

15. Agra IM, Carvalho AL, Pinto CA, Martins EP, Filho JG, Soares FA and Kowalski LP: Biological markers and prognosis in recurrent oral cancer after salvage surgery. Arch Otolaryngol Head Neck Surg 134: 743-749, 2008.

16. Ang KK, Berkey BA, Tu X, Zhang HZ, Katz R, Hammond EH, $\mathrm{Fu}$ KK and Milas L: Impact of epidermal growth factor receptor expression on survival and pattern of relapse in patients with advanced head and neck carcinoma. Cancer Res 62: 7350-7356, 2002.

17. Huang SM, Bock JM and Harari PM. Epidermal growth factor receptor blockade with $\mathrm{C} 225$ modulates proliferation, apoptosis and radiosensitivity in squamous cell carcinomas of the head and neck. Cancer Res 59: 1935-1940, 1999.

18. Zhang N, Erjala K, Kulmala J, Qiu X, Sundvall M, Elenius K and Grénman R: Concurrent cetuximab, cisplatin and radiation for squamous cell carcinoma of the head and neck in vitro. Radiother Oncol 92: 388-392, 2009.

19. Vermorken JB, Herbst RS, Leon X, Amellal N and Baselga J: Overview of the efficacy of cetuximab in recurrent and/or metastatic squamous cell carcinoma of the head and neck in patients who previously failed platinum-based therapies. Cancer 112: 2710-2719, 2008

20. Hasegawa T, Tanakura M, Takeda D, Sakakibara A, Akashi M, Minamikawa $\mathrm{T}$ and Komori T: Risk factors associated with distant metastasis in patients with oral squamous cell carcinoma. Otolaryngol Head Neck Surg 152: 1053-1060, 2015.

21. Sumioka S, Sawai NY, Kishino M, Ishihama K, Minami M and Okura M: Risk factors for distant metastasis in squamous cell carcinoma of the oral cavity. J Oral Maxillofac Surg 71: 1291-1297, 2013.

22. Bulut OC, Lindel K, Hauswald H, Brandt R, Klauschen F, Wolf J, Wolf T, Plinkert PK, Simon C, Weichert W and Stenzinger A: Clinical and molecular characteristics of HNSCC patients with brain metastases: A retrospective study. Eur Arch Otorhinolaryngol 271: 1715-1722, 2014.

23. Ruzevick J, Olivi A and Westra WH: Metastatic squamous cell carcinoma to the brain: An unrecognized pattern of distant spread in patients with HPV-related head and neck cancer. J Neurooncol 112: 449-454, 2013. 
24. Ang KK, Zhang Q, Rosenthal DI, Nguyen-Tan PF, Sherman EJ, Weber RS, Galvin JM, Bonner JA, Harris J, El-Naggar AK, et al: Randomized phase III trial of concurrent accelerated radiation plus cisplatin with or without cetuximab for stage III to IV head and neck carcinoma: RTOG 0522. J Clin Oncol 32: 2940-2950, 2014.

25. Touma W, Koro SS, Ley J, Wildes TM, Michel L, Tao Y and Adkins D: Risk factors for and pre-medications to prevent cetuximab-induced infusion reactions in patients with squamous cell carcinoma of the head and neck. Oral Oncol 50: 895-900, 2014.

26. Chung CH, Mirakhur B, Chan E, Le QT, Berlin J, Morse M, Murphy BA, Satinover SM, Hosen J, Mauro D, et al: Cetuximab-induced anaphylaxis and $\operatorname{IgE}$ specific for galactose-alpha-1,3-galactose. N Engl J Med 358: 1109-1117, 2008.

27. Commins SP, James HR, Kelly LA, Pochan SL, Workman LJ, Perzanowski MS, Kocan KM, Fahy JV, Nganga LW, Ronmark E, et al: The relevance of tick bites to the production of IgE antibodies to the mammalian oligosaccharide galactose- $\alpha-1,3$-galactose. J Allergy Clin Immunol 127: 1286-1293.e6, 2011.
28. Commins SP, Satinover SM, Hosen J, Mozena J, Borish L, Lewis BD, Woodfolk JA and Platts-Mills TA: Delayed anaphylaxis, angioedema, or urticaria after consumption of red meat in patients with $\operatorname{IgE}$ antibodies specific for galactose-alpha-1,3-galactose. J Allergy Clin Immunol 123: 426-433, 2009

29. De Vos FY, Driessen CM, Jaspers HC, van Herpen CM and Simons B: Cetuximab-induced pneumonitis in head and neck cancer patient. Oral Oncol 48: e17-e18, 2012.

30. Ishiguro M, Watanabe T, Yamaguchi K, Satoh T, Ito H, Seriu T, Sakata Y and Sugihara K: A Japanese post-marketing surveillance of cetuximab (Erbituxw ${ }^{\circledR}$ ) in patients with metastatic colorectal cancer. Jpn J Clin Oncol 42: 287-294, 2012.

31. Baselga J, Trigo JM, Bourhis J, Tortochaux J, Cortés-Funes H, Hitt R, Gascón P, Amellal N, Harstrick A and Eckardt A: Phase II multicenter study of the antiepidermal growth factor receptor monoclonal antibody cetuximab in combination with platinum-based chemotherapy in patients with platinum-refractory metastatic and/or recurrent squamous cell carcinoma of the head and neck. J Clin Oncol 23: 5568-5577, 2005. 